\title{
PHOTOMETRIC DECOMPOSITION OF MERGERS IN DISK GALAXIES
}

\author{
M. Mezcua ${ }^{1,2,3}$, A.P. Lobanov ${ }^{3,4}$, E. Mediavilla ${ }^{1,2}$, and M. Karouzos ${ }^{5}$ \\ ${ }^{1}$ Instituto de Astrofísica de Canarias (IAC), E-38200 La Laguna, Tenerife, Spain; mmezcua@iac.es \\ ${ }^{2}$ Universidad de La Laguna, Dept. Astrofísica, E-38206 La Laguna, Tenerife, Spain \\ ${ }^{3}$ Max Planck Institute for Radio Astronomy, Auf dem Hügel 69, D-53121 Bonn, Germany \\ ${ }^{4}$ Institut für Experimentalphysik, Universität Hamburg, Luruper Chausse 149, D-22761 Hamburg, Germany \\ ${ }^{5}$ Center for the Exploration of the Origin of the Universe, Seoul National University, Seoul 151-742, Korea
}

\begin{abstract}
Several observational studies and numerical simulations suggest that mergers must contribute to the evolution of galaxies; however, the role that they play is not yet fully understood. In this paper we study a sample of 52 double nucleus disk galaxies that are considered as candidates for a minor merger event. The luminosity of each of the nuclei and their relative separation are derived from a multi-component photometric fit of the galaxies in the Sloan Digital Sky Survey optical images. We find that the nuclei in most of the sources have projected separations $\leq 4 \mathrm{kpc}$. The ratio of nuclear luminosities indicates that most of the systems are likely in the coalescence stage of a major merger. This is supported by the existence of a single galaxy disk in $65 \%$ of the systems studied and the finding of a correlation between nuclear luminosity and host luminosity for the single-disk systems: those sources fitted with as single disk are in a more evolved stage of the merger and present an enhancement of the nuclear luminosity compared to the double-disk systems, as expected from simulations of galaxy mergers. Finally, we identify a sample of 19 double nucleus disk galaxies in which the two nuclei are physically separated by $\leq 1 \mathrm{kpc}$ and constitute thus a sample of sub-kpc binary active galactic nucleus candidates.

Subject headings: Galaxies: evolution - galaxies: interactions - galaxies: nuclei - galaxies: - photometry.
\end{abstract}

\section{Introduction}

A galaxy merger, understood as a pair of galaxies that are gravitationally bound and whose orbits will dynamically decay until their nuclei merge (Toomre \& Toomre 1972), is a process that lasts a few Gyr (e.g., 1-3 Gyr for major mergers with mass ratios $>1: 3)$ and passes through different stages (e.g., Lotz et al. 2008). Numerical simulations show that in major mergers of disk galaxies there is an enhancement of star formation and active galactic nucleus (AGN) activity during the coalescence stage, when the nuclei merge. This occurs 1.5-2 Gyr after the pre-merger stage (e.g., Springel et al. 2005, Lotz et al.|2008) depending on the disks mass and orbital configuration of the system (e.g., Lotz et al.|2008). A time delay of about
0.1-0.5 Gyr may occur between the observed peak of nuclear starburst and the AGN-triggered activity, either due to intrinsic effects related to the AGN triggering mechanism or due to obscuration of the AGN emission by the surrounding gas and dust (e.g., Springel et al. 2005, Hopkins et al. 2008 Schawinski et al. 2010). Possible observational evidence for this phenomenon has been suggested in some radio galaxies (e.g., Emonts et al. 2006 Mezcua et al. 2012). The most pronounced morphological distortions take place also during the coalescence stage (e.g., Di Matteo et al. 2005 Lotz et al. 2008) and are expected to vanish within about $0.5 \mathrm{Gyr}$ after the nuclei merge, in the postmerger stage (e.g., Lotz et al. 2008), when a single remnant galaxy is formed.

In the hierarchical galaxy formation models 
(e.g., Lacey \& Cole 1993, 1994, Volonteri et al. 2003), galaxies grow in a $\Lambda$ cold dark matter $(\Lambda \mathrm{CDM})$ universe in a 'bottom-up' way through multiple mergers. However, the role of mergers in the growth of the stellar mass and size of local massive galaxies is not yet well understood, especially in terms of its empirical aspect, while alternative processes such as secular evolution (e.g., cold gas accretion) can naturally explain the formation of disk galaxies and pseudobulges (e.g., Kormendy \& Kennicutt 2004 Shlosman 2012).

Major mergers have been shown to be responsible for only $\sim 20 \%$ of the mass growth of massive galaxies at $z<1$ (e.g., de Ravel et al. 2009; LópezSanjuan et al. 2010, which is a significant but not dominant fraction. Therefore, other mechanisms such as cold gas accretion or minor mergers must contribute to the mass growth of galaxies. Minor mergers can lead to a significant increase in mass (e.g., from $\sim 25 \%$ at $z \lesssim 1$ to a factor $\sim 2$ at $z<3$; e.g., López-Sanjuan et al. 2011, Bluck et al. 2012) and have been found to be more efficient at increasing galaxy radii than major mergers. A mass increase due to minor mergers of a factor two can lead to a size increase of up to a factor four in radii in massive galaxies at $z<3$ (e.g., Bluck et al. 2012). The factor of size growth in massive galaxies due to minor mergers is found to range between 2 and 5 at $z \lesssim 1-2$ in both observational (e.g., Daddi et al. 2005, Trujillo et al. 2006, Buitrago et al. 2008, Cimatti et al. 2012 Huertas-Company et al. 2013) and theoretical (e.g., Bournaud et al. 2007 Naab et al. 2009: Oser et al. 2012) studies.

The contribution of minor mergers to star formation is also significant (e.g., $\geq 35 \%$ over cosmic time; Kaviraj 2014) and evidence of minor merging events have been often reported in the literature in the form of recent star formation in early-type galaxies (e.g., Kaviraj et al.|2007, 2009 Fernández-Ontiveros et al. 2011).

Additional direct observations of galaxy mergers and especially of the much less empirically explored minor mergers are needed in order to constrain the galaxy evolutionary models. Several catalogs and surveys of merging galaxies with double nuclei exist (e.g., Mazzarella et al. 1991; Mazzarella \& Boroson 1993; Gimeno et al. 2004 . De Propris et al. 2007, Bridge et al. 2010; Lintott et al.|2011; Lambas et al. 2012, Koss et al. 2012 and references therein), while many studies have been aimed at detecting binary nuclei inferred from AGN properties such as double-peaked emission lines (e.g., Liu et al. 2010a, 2010b; McGurk et al. 2011; Woo et al. 2014) or peculiar jet structures (e.g., Lobanov \& Roland 2005, Roland et al. 2008; Mezcua et al. 2011, 2012).

With the aim of studying the role of minor merger processes in disk galaxies in more detail, we analyze in this paper a small sample of double nucleus disk galaxies classified as minor mergers by Gimeno et al. (2004). A photometric point spread function (PSF) fitting is used to estimate the brightness of each of the nuclei and the distance between them in the optical images provided by the Sloan Digital Sky Survey (SDSS) 1 . Based on these fits, we obtain the nuclear luminosities and the separation between the two nuclei in these systems and study their correlation with the host galaxy.

The sample of galaxies analyzed is presented in Section 2. The description of the PSF fitting technique and of the analysis performed is explained in Section 3. The results obtained are presented in Section 4 and discussed in Section 5. Final conclusions are given in Section 6 .

Through this paper, we assume a $\Lambda$ CDM cosmology with parameters $H_{0}=73 \mathrm{~km} \mathrm{~s}^{-1} \mathrm{Mpc}^{-1}$, $\Omega_{\Lambda}=0.73$, and $\Omega_{\mathrm{m}}=0.27$.

\section{The sample}

The sources analyzed are drawn from a catalog of double nucleus disk galaxies suggested as candidates for minor merger events (Gimeno et al. 2004). The catalog comprises 107 double nucleus galaxies selected from older catalogs under the following three requirements: (1) galaxies with redshift $z<0.05$ (or apparent $B$ magnitude $\left.m_{\mathrm{B}}<18\right) ;(2)$ galaxies showing disk-like morphology (i.e., elliptical and $\mathrm{cD}$ galaxies are excluded) to avoid the inclusion of major merger remnants and include only likely minor merger events; (3) galaxies not exhibiting strong tidal distortions or tails, as these features are expected in major merger events (Gimeno et al. 2004).

Of the 107 double nucleus disk galaxies, 60 are found to have imaging data in the SDSS Data Release 8 (SDSS DR8 hereafter; Eisenstein et al.

\footnotetext{
${ }^{1}$ Sloan Digital Sky Survey, www.sdss.org
} 
2011, and references therein). Images of some of these sources in gri colors are shown in Figure 1. FITS images of the 60 objects in the $u, g$, and $r$ bands (centered at $3551 \AA, 4686 \AA$, and $6166 \AA$, respectively) are retrieved by coordinates from the Science Archive Server (SAS) of the SDSS with a size of $2048 \times 1489$ pixels and a pixel scale of $0^{\prime \prime} .396 \mathrm{pixel}^{-1}$. The retrieved images are calibrated and have an applied sky-subtraction appropriate for large objects (see Eisenstein et al. 2011 for a more detailed description).

For the objects MCG +06-07-020 and Mrk 1341, the coordinates reported in Gimeno et al. (2004) do not provide the SDSS field image where these sources should be located. The corrected coordinates are found in $\mathrm{NED}^{2}$ by name and are, for MCG +06-07-020: R.A.(J2000) $=02^{\mathrm{h}} 55^{\mathrm{m}} 29^{\mathrm{s}}$, decl. $(\mathrm{J} 2000)=36^{\circ} 12^{\prime} 0^{\prime \prime} .2$ and for Mrk 1341: R.A. $(\mathrm{J} 2000)=13^{\mathrm{h}} 00^{\mathrm{m}} 59^{\mathrm{s}}$, decl. $(\mathrm{J} 2000)=-00^{\circ} 01^{\prime} 39^{\prime \prime}$.

\section{Data analysis}

\subsection{PSF fitting}

The analysis of the images is in a first step attempted using the IRAF ${ }^{3}$ software and the PSF fitting code imfitfits (McLeod et al. 1998), which fits a model convolved with a PSF image. The model is optimized by computing the $\chi^{2}$ to fit the observed image. In order to determine the brightness of each individual nucleus, the field images are first cropped to a size of $64 \times 64$ pixels that includes the two nuclei and the host galaxy of each target. An isolated, bright, but not saturated (less than 15,000 counts at the peak) star is chosen from the field image and a region of 64 $\times 64$ pixels is cut around it. Using this star image as the PSF, we attempt a two-dimensionalmulticomponent fitting of the central region where the two nuclei lie. In principle, we may have attempted a fit based on the canonical galaxy components: one or two galaxy disks, one or two bulges, and two nuclei. However, the spatial resolution is not sufficient to identify the slope changes at the inner central regions and the use of three components yields a degeneracy in the solutions.

\footnotetext{
${ }^{2}$ NASA/IPAC Extragalactic Database.

${ }^{3}$ IRAF is distributed by the National Optical Astronomy Observatories operated by the Association of Universities for Research in Astronomy, Inc. under cooperative agreement with the National Science Foundation.
}

In addition, it is not clear whether such physical decomposition applies to minor mergers where the two nuclei are present. We thus decide to adopt a less ambitious approach and distinguish only between compact and extended components by using only two components per merger system: two PSFs for the nuclei, and one or two exponential disks for the galaxy or galaxies. This should provide statistically reasonable estimates of the intensities of the compact components and good measurements of the nuclear distances, while reducing the degeneracy as much as possible.

Using this decomposition we are able to perform satisfactory two-dimensional fittings to $50 \%$ of the double nucleus galaxies in our sample. The uncertainty of the nuclear intensities fitted using imfitfits can be estimated from the residual image created after subtraction of the model image from the data (see Figure 2). We obtain an uncertainty $\leq 10 \%$, which supports the consistency of the photometric decomposition. In one case (Mrk 22) we need to consider a de Vaucouleurs profile to fit the host. In order to visually check the goodness of the fits, we extract a one-dimensional vector along the position of the nuclei and subtract the model fit from the observed profile to visualize the residuals (see Figure 3).

For those galaxies whose luminosity profiles are not well-fitted using a two-dimensional photometric model, a one-dimensional vector is extracted along the position of the nuclei using the IRAF task pvector. These one-dimensional profiles are then fitted with Gaussian components using the Starlink DIPSO software package (Howarth \& Murray 1991). A Gaussian of fixed width is fitted to each of the nuclei (the width is constrained to a value $\simeq$ to the stellar PSF), while a Gaussian of variable width is used to fit the host galaxy. In some cases, two Gaussians are required to fit two host galaxy disks (see Figure 4). The errors of the nuclear intensities provided by DIPSO are $\leq 20 \%$. To explore the systematic errors due to the two different photometric models used, we compare the twodimensional fitting of imfitfits with the onedimensional results from DIPSO for those galaxies that could be fitted by the two models. The difference between the two models is going to be a conservative estimate of the systematic errors induced by the photometric modeling. We find that the differences in nuclear intensities are $\leq 30 \%$. 

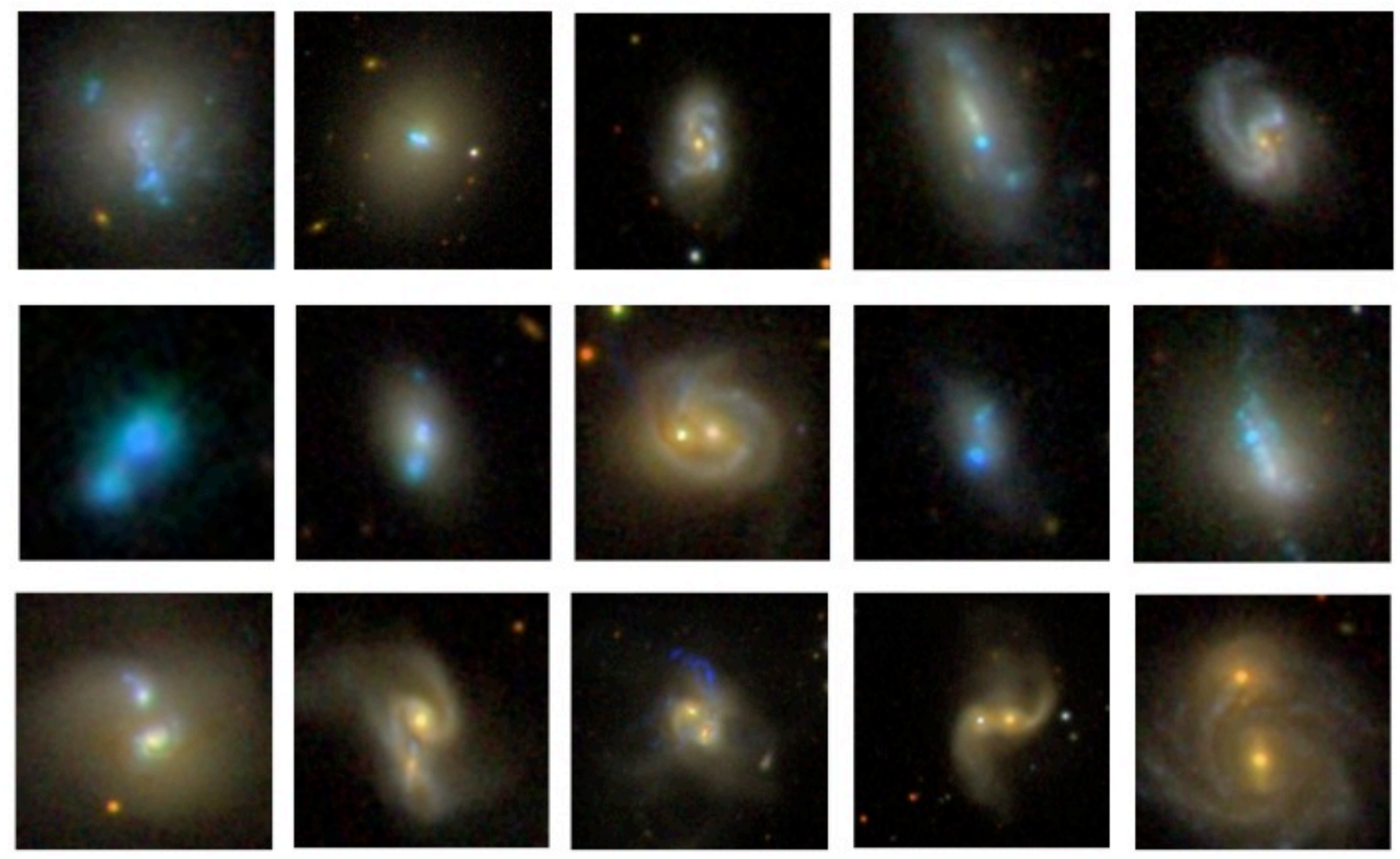

Fig. 1.- From top to bottom, left to right: color gri SDSS DR8 images of the sources NGC 5058, NGC 3773, Mrk 1114, Mrk 712, Mrk 721, Mrk 116, Mrk 104, NGC 3758, Mrk 1263, NGC 7468, NGC 5860, Mrk 423, NGC 5256, Mrk 212, and MCG +00-12-073. Sources are ordered in ascending nuclear separation. The field of view is different for each object (i.e., $25 \operatorname{arcsec} \times 25 \operatorname{arcsec}, 51 \operatorname{arcsec} \times 51 \operatorname{arcsec}$, and $100 \operatorname{arcsec}$ $\times 100 \mathrm{arcsec}$ ) so that the morphological type of the host galaxy can be appreciated.

(A color version of this figure is available in the online journal.)
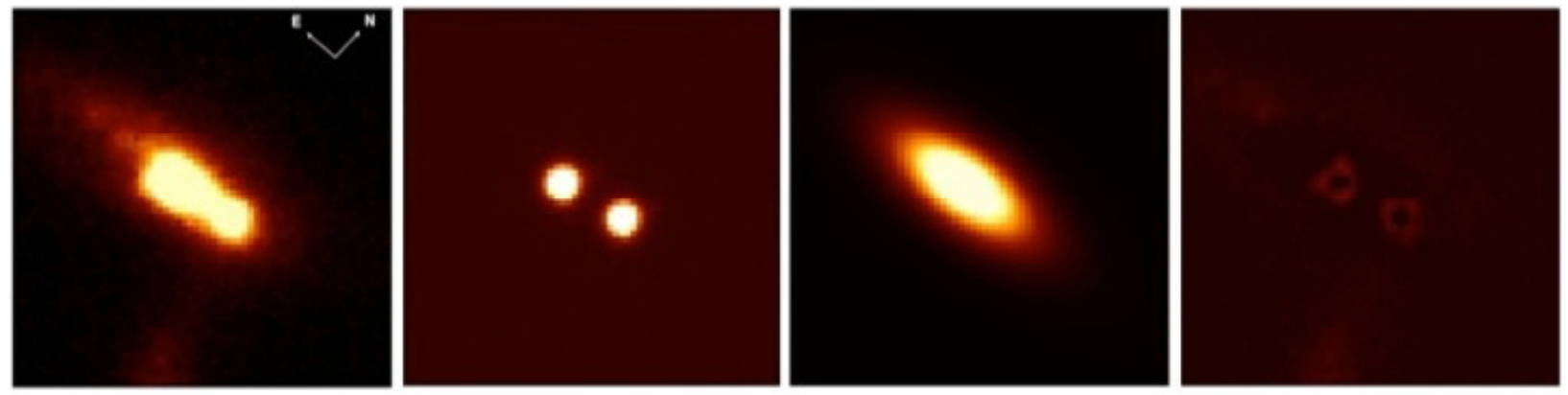

Fig. 2.- PSF fitting of Mrk 19. Figures from left to right show the observed $r$-band image from SDSS DR8, the model image of the two nuclei fitted by imfitfits, the model image of the host galaxy fitted by imfitfits, and the residual image. The image sizes are 25 arcsec $\times 25$ arcsec.

(A color version of this figure is available in the online journal.) 

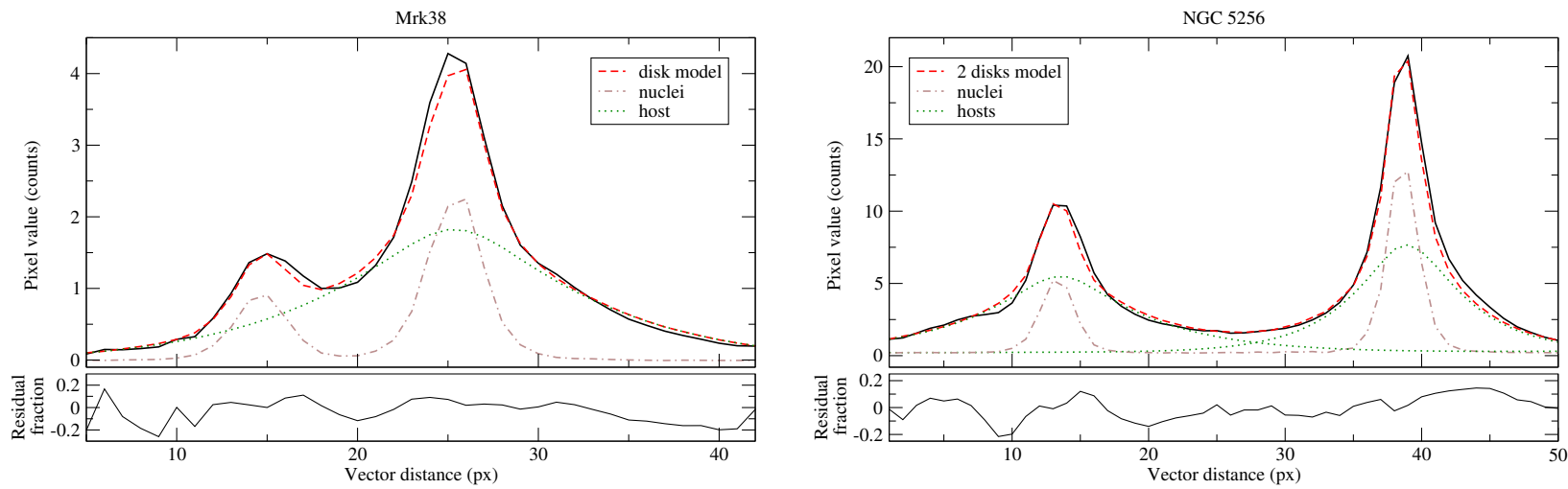

Fig. 3.- One-dimensional vector (solid black line) extracted along the position of the nuclei of Mrk 38 (left) and NGC 5256 (right). The two-dimensional imfitfits photometric fit of a disk (for Mrk 38) and 2 disks (for NGC 5256) is shown (red dashed line) decomposed into the host fit (green dotted line) and the fit to the two nuclei (brown dotted-dashed line). The fractional residuals are shown at the bottom of each plot. (A color version of this figure is available in the online journal.)
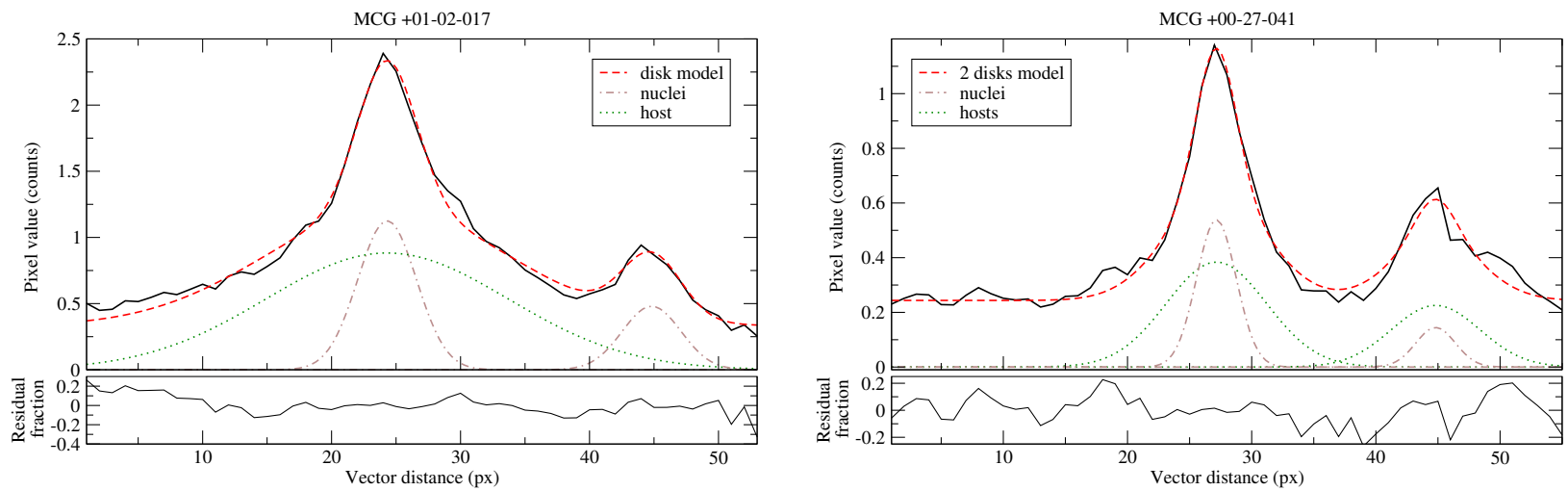

Fig. 4.- One-dimensional DIPSO photometric fit of MCG +01-02-017 (left) and MCG +00-27-041 (right). As in the previous plot, the lines plotted are: profile (solid black line), photometric fit (red dashed line), host galaxy fits (green dotted line), and fit of the two nuclei (brown dotted-dashed line). One disk model is needed to fit the host galaxy of MCG +01-02-017, while two disks are required to fit the host of MCG $+00-27-041$. The fractional residuals are shown at the bottom of each plot.

(A color version of this figure is available in the online journal.) 
The error in the relative separation between the two nuclei is derived as the quadratic sum of the astrometric error of each nucleus (provided by the global astrometric precision of the SDSS DR8, which is 0.1 arcsec) and the uncertainty of the photometric fit (we use conservative limits of $10 \%$ for the sources fitted with imfitfits and $30 \%$ for the ones fitted with DIPSO).

\subsection{Nuclear and Host Galaxy Luminosi- ties}

The intensities obtained from imfitfits for each of the two nuclei and host galaxies are converted to magnitudes using the calibration specified in the header of each SAS image. We correct these magnitudes for Galactic dust extinction using the reddening corrections at the position of each object provided by SDSS (following Schlegel et al. 1998) and convert them to absolute AB magnitudes (Oke \& Gunn 1983). The $r$-band luminosities of the nuclei are then derived using $L / L_{\odot}=10^{\left(M-M_{\odot}\right) / 2.5}$, where $M$ is the absolute magnitude of the target, and $M_{\odot}$ and $L_{\odot}$ are, respectively, the absolute $r$-band magnitude and luminosity of the Sun. Their values, used here, are $M_{\odot}=4.42$ and $L_{\odot}=3.85 \times 10^{26} \mathrm{~W}$ (e.g., Phillips 1995; Binney \& Merrifield 1998).

\section{Results}

The results of the PSF fitting (i.e., magnitudes and luminosities of the primary and secondary nucleus and the host galaxy, and the relative separation between the nuclei) together with the name and distances of the galaxies analyzed are listed in Table 1. Of the 60 objects for which SDSS DR8 images are available, three do not have any spectroscopical identification nor redshift available and thus their nuclear and host galaxy luminosities could not be derived. These objects are therefore excluded from the analysis and are not included in Table 1. The fitting codes are not able to find a double nucleus in three other targets, which are thus also removed from the analysis. The host galaxy of two other sources, MCG +06-21-031 and MCG +10-21-040, could neither be fitted by a twodimensional nor by a one-dimensional model as the SDSS images were very noisy. These two sources are also excluded from the analysis. Therefore, the total number of objects included in Table 1 and considered for further analysis is 52 .

\subsection{Extinction}

To ensure that the results obtained are not affected by different degrees of extinction in each of the nuclei, the $u$ and $g$-band images of the 52 target sources in Table 1 are retrieved from the SDSS DR8 and PSF fitting with imfitfits is performed in these bands. The images are first cropped to 64 $\times 64$ pixels and a point source model is fitted to each of the nuclei using a stellar image as PSF. The parameters of the model are the positions and intensities of each of the nuclei and the background level of the image. The steps are the same as described in Section 3 . In the $u$ band, imfitfits fails to fit the positions of the double nuclei in nine of the targets. For the remaining 43 sources, the fitted fluxes are converted from nanomaggies to magnitudes and the $u-g$ colors of the primary against the secondary nucleus are plotted in Figure 5. The figure shows that both nuclei seem to be in general affected by the same extinction, indicating that they both have the same environment. The results obtained in the previous sections (Table 1) are thus not affected by differences in the ambient medium in which the nuclei are embedded.

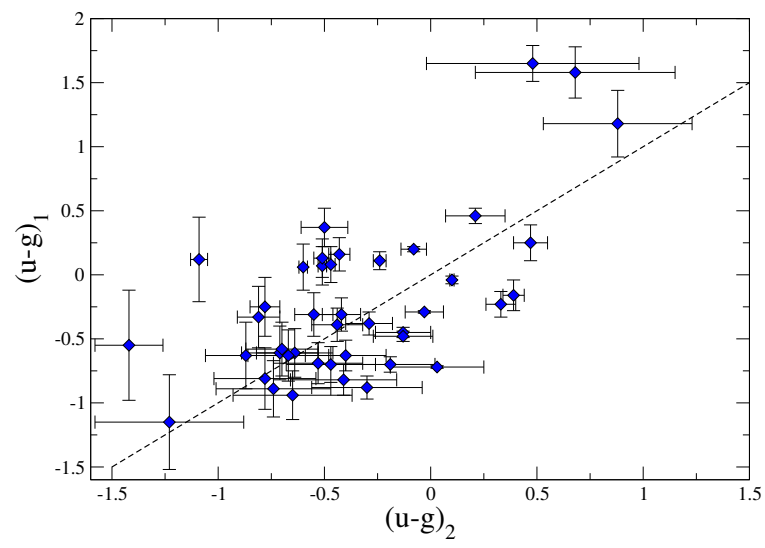

Fig. 5.- Color-color diagram of $u-g$ magnitudes of the primary vs. the $u-g$ magnitudes of the secondary nucleus. A one-to-one correlation indicating no systematic difference in extinction between the two nuclei is also plotted (dashed line).

(A color version of this figure is available in the online journal.) 
We also derive the amount of extinction allowed by the fit of the nuclear luminosities. Within the $30 \%$ error of the nuclear intensities, an extinction of $A_{r} \leq 0.3 \mathrm{mag}$ is allowed. A higher degree of extinction might be present in these double nucleus galaxies, where nuclear star-formation might be contributing to the observed nuclear intensities (see Section 5).

\subsection{Major and Minor Mergers}

The target sources were classified as minor merger candidates based on their morphological appearance lacking strong tidal tails (Gimeno et al. 2004). However, the lack of these features can also indicate that some sources might be in a late stage of a major merger event when the optical morphological distortions have already vanished and are only recognizable in studies of the neutral hydrogen (HI) gas (e.g., Emonts et al. 2006, Koribalski \& López-Sánchez 2009). In the case of very shallow imaging, like in the standard-depth SDSS images analyzed here, the lack of visible morphological distortions results from the very short exposure times (i.e., of only $53.9 \mathrm{~s}$ per band), which are insufficient for revealing the tidal features owing to their faintness compared to the host galaxy magnitude. To detect tidal features around mergers (especially for minor mergers) much deeper imaging is required (e.g., deep Stripe82 images; Kaviraj 2010, see also van Dokkum 2005, Sheen et al. 2012).

To determine what is the stage of the merger event and whether morphological features should be present, we derive the ratio of nuclear luminosities $L_{2} / L_{1}$, usually adopted to classify sources into major and minor mergers (e.g., Lambas et al. 2012 ). We adopt a luminosity ratio $>1: 3$ for major mergers. We obtain that only $38 \%$ of the target sources (20 out of 52) qualify as minor mergers, while most of the sources studied $(62 \%)$ are classified as major mergers. Since these sources exhibit two nuclei, most of these systems must be in the coalescence stage of a major merger in which the nuclei are in the process of merging but have not yet merged and a single galaxy with morphological distortions is formed (e.g., Springel et al. 2005; Lotz et al. 2008). The non-detection of these strong tidal features is thus caused by the low exposure time of the SDSS images. The finding that most of the galaxies studied qualify as major mergers despite their initial morphological classification as minor mergers (Gimeno et al. 2004) is of significant importance for studies based on visual classification of galaxies, as many so-called "minor mergers" might actually be mis-classifications of major mergers.

\subsection{The Coalescence Stage}

We find that in 19 out of the 52 sources the two nuclei are separated by a projected distance $\leq 1 \mathrm{kpc}$ (see Figure 6). A secondary peak in the distribution is observed for objects with nuclear projected separations between 2 and $3 \mathrm{kpc}$. A total of $91 \%$ of the sources have nuclear projected separations $\leq 4 \mathrm{kpc}$, which is in agreement with the results from Gimeno et al. (2004). As suggested by these authors, the lower number of double nucleus galaxies with larger nuclear separations may be a selection effect: the greater the distance between the host galaxy and the merging satellite, the less recognizable is the merger system as a double nucleus galaxy. The double nucleus galaxies with the shortest nuclear separations would thus belong to an evolved stage of the merger (i.e., the coalescence phase) in which a single galaxy has already been formed. This is supported by the finding that $65 \%$ (33 out of $511^{4}$ ) of the sources were fitted by one disk host galaxy, which indicates that the disk of the satellite galaxy is no longer recognizable and is in agreement with the finding that $62 \%$ of the target sources qualify as major mergers according to the ratio of nuclear luminosities (see Section 4.2). On the other hand, the main peak of 19 sources with nuclear separation $\leq 1 \mathrm{kpc}$ (Figure 6) is mainly formed by the major mergers systems, while the secondary peak in the range $2-3 \mathrm{kpc}$ is owing to the peak in the distribution of minor mergers. These results yield further support to the suggestion that those systems with the shortest nuclear distances must be in the coalescence stage of a major merger.

\section{Discussion}

\subsection{The Nature of the Nuclei}

Identifying the energy source of the nuclei studied here is not trivial. We have shown that, for the

\footnotetext{
${ }^{4}$ The fit of one source required no host galaxy disks.
} 


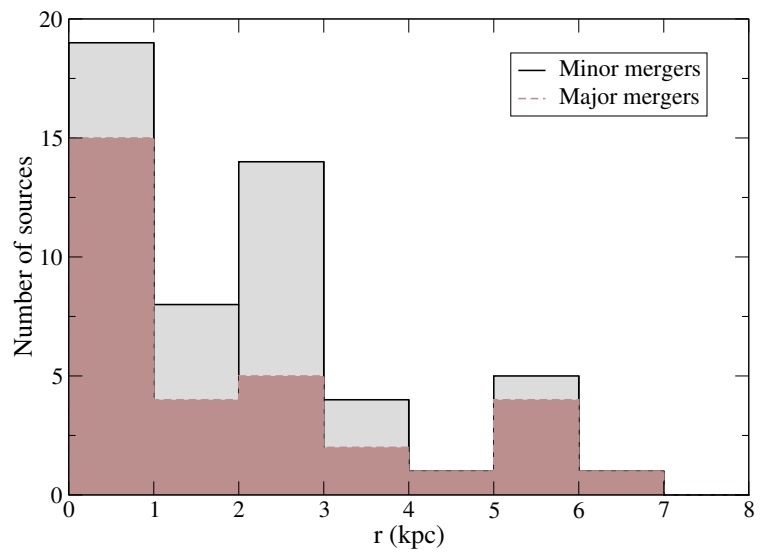

Fig. 6.- Distribution of projected separations between the two nuclei for sources classified as major mergers (dashed line, red bars) and minor mergers (solid line, gray bars).

(A color version of this figure is available in the online journal.)

majority of these sources, the scenario of a coalescence phase in a major merger is more plausible rather than that of an ongoing minor merger. For these sources, the presence of a black hole $(\mathrm{BH})$ in each of the two nuclei is expected. This $\mathrm{BH}$ can either be actively accreting or be dormant. Conversely, for those sources identified as minor mergers, the secondary nucleus can potentially in addition be either a star-forming region or a stellar cluster. To test these different possibilities, we investigate whether these sources are detected in the mid-IR (MIR, by the Wide-field Infrared Survey Explorer, (WISE); Wright et al. 2010) or the farUV (by the Galaxy Evolution Explorer (GALEX); Martin et al. 2005). Given the small angular separation of the two nuclei, they cannot be resolved by WISE nor GALEX in almost all the galaxies of our sample. However, the WISE colors of the 52 sources reveal that 2 of them $(\sim 4 \%)$, MCG +00-04-098 and NGC 3758, have AGN-like colors (e.g., Stern et al. 2012, Mateos et al. 2012), while the rest occupy the star-formation region of the plot. These results suggest that star formation rather than AGN dominates the emission in the majority of the sample, although the presence of a deeply embedded AGN cannot be excluded. Such highly obscured AGN are expected during the process of disk-galaxy major mergers (e.g., Sanders et al. 1988, Canalizo \& Stockton 2001). In that case, higher-resolution MIR observations are required to distinguish between AGN and star formation by separating the nuclear and the extended MIR contribution, as is the case of Mrk 789, which is known to host a Seyfert nuclei (e.g., Ramos Almeida et al. 2007) despite its location outside the AGN locus in the WISE color diagram.

The presence of an AGN can be also confirmed by hard X-ray observations (if the X-ray emission is Compton thin) with high angular resolution (with the Chandra X-ray satellite) in the case of binary AGN at kpc scales. Several kpc-scale binary AGNs have been found using X-ray observations (e.g., Komossa et al. 2003, Guainazzi et al. 2005, Hudson et al. 2006; Bianchi et al. 2008; Comerford et al. 2011; Koss et al. 2011). The closest separations in an AGN pair have so far been detected in major mergers of spiral galaxies (NGC 6240, projected separation $\sim 1 \mathrm{kpc}$; Komossa et al. 2003, and Mrk 739, projected separation $\sim 3.4 \mathrm{kpc}$; Koss et al. 2011), setting strong constrains on the co-evolution of galaxies and their nuclear black holes. The occurrence of minor mergers should be more common; however, only one AGN pair has been found in a merger of unequal mass galaxies (NGC 3393, projected separation $\sim 150$ pc; Fabbiano et al. 2011). In the total sample of 52 double nucleus systems, the PSF fitting finds 19 sources with projected nuclear separations $\leq 1.0 \mathrm{kpc}$, or physical separations $\leq 1.1$ $\mathrm{kpc}$ when considering an average inclination angle for the host galaxies of $\sim 69^{\circ}$ (Gimeno et al. 2004). Most of the 19 sub-kpc pairs qualify as major mergers (15 sources), while 4 of the sources are classified as minor merger events (see Section 4.3). We have searched for any Chandra X-ray studies or observations available in the literature and the archives of the 52 sources analyzed in this paper. We find that three sources have been studied in detail with Chandra and that in two of them (NGC 5256, Brassington et al. 2007; NGC 3758, Koss et al. 2011) the presence of a binary AGN is confirmed. The nuclear separations obtained from the SDSS photometric fit (6.2 arcsec for NGC 3758 and 10.2 arcsec for NGC 5256, see Table 1) are consistent with the separations derived from the Chandra observations. NGC 5256 qualifies as a 
major merger according to the ratio of nuclear luminosities, in agreement with the classification of Brassington et al. 2007, while NGC 3758 is classified as a minor merger. Archival Chandra data are available for four more sources, whose analysis is left for future work.

Finally, nuclear accretion within the galaxy pairs could also be probed from their location in emission-line diagnostic diagrams, although, as seen above, star formation can in some cases overwhelm the AGN emission, whose presence can only by definitely confirmed by hard X-ray observations. This is the case, for instance, for NGC 3758, which was confirmed as a binary AGN from Chandra observations although the secondary nucleus does not qualify as an AGN based on its optical emission lines (Koss et al. 2011).

Of particular interest is the case of the presence of a low-mass black hole in the satellite galaxy nucleus of those 20 sources that we identified as minor mergers. Low-mass BHs (or intermediatemass BHs, IMBHs) are expected in the nuclei of low-mass dwarf galaxies from the BH-mass scaling relationships (e.g., McConnell \& Ma 2013). The tidal stripping of merging satellite low-mass galaxies should thus yield the presence of IMBHs in the haloes of galaxies (Volonteri \& Madau 2008, Bellovary et al. 2010). Several IMBH candidates have already been found from X-ray and radio studies of ultraluminous X-ray sources (e.g., Farrell et al. 2009, Webb et al. 2012, Mezcua \& Lobanov 2011, Mezcua et al. 2013 a, 2013b). However, their presence in the nucleus of captured satellite galaxies still remains an open question.

\subsection{The Nucleus-Host Connection}

The coalescence phase of a merger is seen in simulations to coincide with the peak of mergerinduced star-formation and AGN activity (e.g., Springel et al. 2005, Lotz et al. 2008, Hopkins et al. 2008), therefore an increase in nuclear luminosity is expected in the coalescence stage.

The detection of both galaxies in the doubledisk systems implies an earlier stage of the merging process (i.e., between the first passage and the coalescence stages). In these systems some enhancement of the host galaxy luminosity is expected due to galaxy-wide merger-triggered star formation. Conversely, the single-disk systems are at a more evolved stage of the merger (i.e., the coalescence stage), when the two nuclei should have sunk well into the gravitational center of the merging system. At this stage, single-disk systems should exhibit enhanced nuclear luminosities compared to the double-disk galaxies, caused by either the starburst or the AGN merger-triggered activity. We investigate this by plotting the nuclear luminosity of the primary nucleus versus the host galaxy luminosity (Figure 7).

The trends observed for the two sub-samples of sources (i.e., the single-disk and double-disk systems) are significantly different. We perform a linear fit and correlation analysis separately for each sub-sample. We find a slope of $0.77 \pm 0.01$ for the single-disk systems (red circles in Figure 7) with a Pearson's correlation coefficient, $\rho$, of 0.9. Although our sample is at very low redshifts $(z<0.05)$, we still expect some degree of inter-dependence between the host and nuclear luminosities due to redshift. We calculate the partial correlation coefficient considering this redshift dependence, $\rho_{p}$, and find it to be 0.8 for the singledisk sub-sample. Turning now to the double-disk systems (blue triangles in Figure 7), we calculate a slope of $1.22 \pm 0.18$ with $\rho=0.8$ and $\rho_{p}=0.4$. The large $\sigma$ value of the slope and the low significance of the correlation as indicated by $\rho_{p}$ imply that, unlike for the single-disk sources, the loose correlation observed for the double-disk systems is mainly due to redshift effects. This indicates that the host galaxy luminosity for the doubledisk sources does not or only weakly follows the luminosity of the nucleus.

We note that the slope of the linear regression for the single-disk galaxies is steeper than that of the double-disk systems. When considering the systems with two identifiable disks (blue triangles in Figure 7) at luminosities $<10^{41} \mathrm{erg} \mathrm{s}^{-1}$, we find that they appear to lie exclusively below the trend observed for the single-disk sources. These findings are in line with the theoretical expectations for single-disk systems described above. For the double-disk systems we expect that at later times (and at smaller separations when the two disks will have merged) the nucleus will also brighten up, putting these sources back on the implied trend.

High luminosity $\left(>10^{41}\right.$ erg s $^{-1}$ ) sources with two identifiable host galaxies do not show the nuclear luminosity deficit observed for their lower lu- 
minosity counterparts, following the trend implied by the single-disk sources (within $\sim 1 \sigma$ ). These sources might be at an earlier phase of the merger, plausibly having yet to experience the enhancement of star-formation and AGN activity. This conclusion is corroborated by the fact that all five sources exhibit rather large separations between their two nuclei $(r>4 \mathrm{kpc})$, occupying the high end of the nuclear separation distribution of the whole sample.

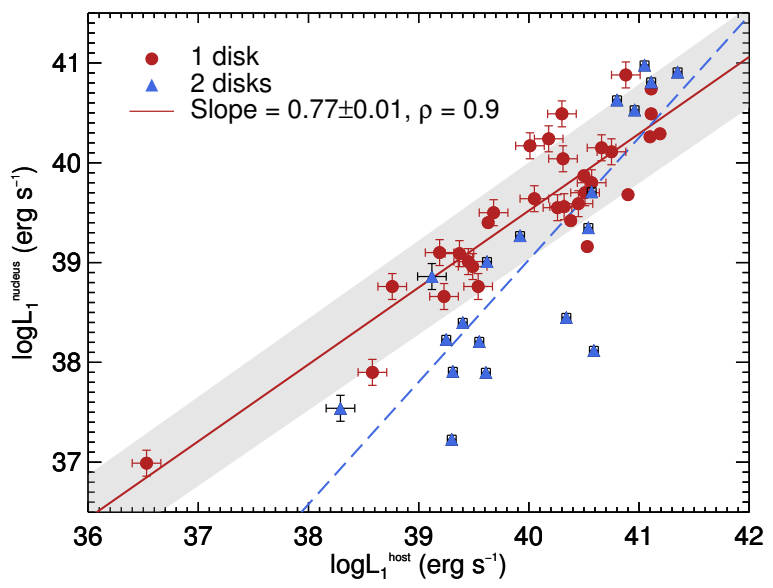

Fig. 7.- Luminosity of the primary nucleus vs. host luminosity. Sources with one (red circles) and two (blue triangles) fitted disk host galaxies are plotted. A linear fit to the data for single-disk sources (red solid line) and double-disk sources (blue dashed line) is plotted. We also plot the $1 \sigma$ uncertainty (gray shaded area) of the fitted slope for the single-disk systems. The slope and Pearson's correlation coefficient of this linear fit are also shown.

(A color version of this figure is available in the online journal.)

As reported in Section 4.3, 65\% of the sources studied are under the process of a major merger as implied by their nuclear luminosity ratios and number of host galaxies fitted. The result of the major merger of two disk galaxies is a spheroidal galaxy characterized by the presence of a bulge or pseudo-bulge (e.g., Cox et al. 2006; Hopkins et al. 2010: Keselman \& Nusser 2012). The evolution of supermassive BHs (SMBHs) at the centers of bulge and pseudo-bulge galaxies has been found to be closely related to host galaxy properties such as the stellar velocity dispersion (e.g., Ferrarese \& Merritt 2000; Gebhardt et al. 2000 Graham et al. 2011) or the bulge luminosity (e.g., $M_{\mathrm{BH}} \propto$ $L_{\text {bulge }}^{\text {0.90 }}$ Wandel 2002; $M_{\mathrm{BH}} \propto L_{\text {bulge }}^{0.80 \pm 0.09}$, Bentz et al. 2009).

As shown in Figure 7, the linear regression analysis shows a clear positive correlation between $L_{1}$ and $L_{\text {host1 }}$ of slope $0.77 \pm 0.01$ for the singledisk systems but not for the double-disk galaxies. Given the presence of an actively accreting SMBH within each nucleus, the correlation between the luminosity of the primary nucleus and the host galaxy luminosity, although shallower, seems to be analogous to the $M_{\mathrm{BH}} \propto L_{\text {bulge }}^{0.9}$ correlation of scatter 0.1 dex derived both for inactive and active galaxies (Wandel 2002, see also Marconi \& Hunt 2003 Bentz et al. 2009). This correlation is not expected in disk galaxies but only in systems with bulges or pseudo-bulges. Its emergence for merging systems with one host disk, but not with two host disks, can be explained by them qualifying as major mergers in which the putative SMBHs are or will go on to form a bulge or pseudobulge and eventually fall on the $M_{\mathrm{BH}} \propto L_{\text {bulge }}$ correlation.

\section{Conclusions}

In this paper, we have performed a photometric PSF fitting for a sample of double nucleus disk galaxies candidates for minor merger. This has enabled estimating the luminosity of the primary nucleus, the secondary nucleus, and the host galaxy of 52 double nucleus disk galaxies and the distance between the two nuclei. Despite the initial classification of these sources as minor mergers, we find that most of the targets qualify as major mergers based on the ratio of nuclear luminosities (32 out of 52 sources) and on the finding that $65 \%$ of the host galaxies are fitted by one exponential disk. These sources must therefore be in a more evolved stage of the merger (i.e., the coalescence stage) than the double-disk systems. We find that $\sim 90 \%$ of the sources studied have projected nuclear separations $\leq 4 \mathrm{kpc}$, of which in 19 sources the two nuclei are separated by $\leq 1 \mathrm{kpc}$. There are very few confirmed cases of kpc-scale binary AGN, therefore these 19 sources are potential binary AGN candidates to be targeted by high-resolution Xray observations. Finally, we present evidence for 
the systems fitted with one host to show enhancement of their nuclear luminosity as expected from simulations. This is possibly caused by the peak of the merger-triggered star-formation and AGN activity.

\section{Acknowledgments}

The authors are grateful for the suggestions of the anonymous referee that helped to significantly improve the manuscript. The authors are very thankful to Joe Mazzarella and Richard Davies for insightful discussions. M.M. was supported for this research through a stipend from the International Max Planck Research School (IMPRS) for Astronomy and Astrophysics at the Universities of Bonn and Cologne. M.K. was supported for this research by the National Research Foundation of Korea (NRF) grant, No. 2008-0060544, funded by the Korea government (MSIP). Part of this work was supported by the COST Action MP0905 "Black Holes in a Violent Universe".

\section{REFERENCES}

Bellovary J. M., Governato F., Quinn T. R., Wadsley J., Shen S., Volonteri M., 2010, ApJ, 721, L148

Bentz M. C., Peterson B. M., Pogge R. W., Vestergaard M., 2009, ApJ, 694, L166

Bianchi S., Chiaberge M., Piconcelli E., Guainazzi M., Matt G., 2008, MNRAS, 386, 105

Binney J., Merrifield M., 1998, Galactic Astronomy

Bluck A. F. L., Conselice C. J., Buitrago F., Grützbauch R., Hoyos C., Mortlock A., Bauer A. E., 2012, ApJ, 747, 34

Bournaud F., Jog C. J., Combes F., 2007, A\&A, 476,1179

Brassington N. J., Ponman T. J., Read A. M., 2007, MNRAS, 377, 1439

Bridge C. R., Carlberg R. G., Sullivan M., 2010, ApJ, 709, 1067

Buitrago F., Trujillo I., Conselice C. J., Bouwens R. J., Dickinson M., Yan H., 2008, ApJ, 687, L61
Canalizo G., Stockton A., 2001, ApJ, 555, 719

Cimatti A., Nipoti C., Cassata P., 2012, MNRAS, 422, L62

Comerford J. M., Pooley D., Gerke B. F., Madejski G. M., 2011, ApJ, 737, L19

Cox T. J., Dutta S. N., Di Matteo T., Hernquist L., Hopkins P. F., Robertson B., Springel V., 2006, ApJ, 650, 791

Daddi E. et al., 2005, ApJ, 626, 680

De Propris R., Conselice C. J., Liske J., Driver S. P., Patton D. R., Graham A. W., Allen P. D., 2007, ApJ, 666, 212

de Ravel L. et al., 2009, A\&A, 498, 379

Di Matteo T., Springel V., Hernquist L., 2005, Nature, 433, 604

Eisenstein D. J. et al., 2011, AJ, 142, 72

Emonts B. H. C., Morganti R., Tadhunter C. N., Holt J., Oosterloo T. A., van der Hulst J. M., Wills K. A., 2006, A\&A, 454, 125

Fabbiano G., Wang J., Elvis M., Risaliti G., 2011, Nature, 477, 431

Farrell S. A., Webb N. A., Barret D., Godet O., Rodrigues J. M., 2009, Nature, 460, 73

Fernández-Ontiveros J. A., López-Sanjuan C., Montes M., Prieto M. A., Acosta-Pulido J. A., 2011, MNRAS, 411, L21

Ferrarese L., Merritt D., 2000, ApJ, 539, L9

Gebhardt K. et al., 2000, ApJ, 539, L13

Gimeno G. N., Díaz R. J., Carranza G. J., 2004, AJ, 128, 62

Graham A. W., Onken C. A., Athanassoula E., Combes F., 2011, MNRAS, 412, 2211

Guainazzi M., Piconcelli E., Jiménez-Bailón E., Matt G., 2005, A\&A, 429, L9

Hopkins P. F. et al., 2010, ApJ, 715, 202

Hopkins P. F., Hernquist L., Cox T. J., Kereš D., 2008, ApJS, 175, 356 
Howarth I. D., Murray J., 1991, SERC-RAL Starlink User Note 50.13

Hudson D. S., Reiprich T. H., Clarke T. E., Sarazin C. L., 2006, A\&A, 453, 433

Huertas-Company M. et al., 2013, MNRAS, 428, 1715

Kaviraj S., 2010, MNRAS, 406, 382

Kaviraj S., 2014, MNRAS, 437, L41

Kaviraj S., Peirani S., Khochfar S., Silk J., Kay S., 2009, MNRAS, 394, 1713

Kaviraj S. et al., 2007, ApJS, 173, 619

Keselman J. A., Nusser A., 2012, MNRAS, 424, 1232

Komossa S., Burwitz V., Hasinger G., Predehl P., Kaastra J. S., Ikebe Y., 2003, ApJ, 582, L15

Koribalski B. S., López-Sánchez Á. R., 2009, MNRAS, 400, 1749

Kormendy J., Kennicutt Jr. R. C., 2004, ARA\&A, 42,603

Koss M. et al., 2011, ApJ, 735, L42

Koss M., Mushotzky R., Treister E., Veilleux S., Vasudevan R., Trippe M., 2012, ApJ, 746, L22

Lacey C., Cole S., 1993, MNRAS, 262, 627

Lacey C., Cole S., 1994, MNRAS, 271, 676

Lambas D. G., Alonso S., Mesa V., O'Mill A. L., 2012, A\&A, 539, A45

Lintott C. et al., 2011, MNRAS, 410, 166

Liu X., Greene J. E., Shen Y., Strauss M. A., 2010a, ApJ, 715, L30

Liu X., Shen Y., Strauss M. A., Greene J. E., 2010b, ApJ, 708, 427

Lobanov A. P., Roland J., 2005, A\&A, 431, 831

López-Sanjuan C., Balcells M., Pérez-González P. G., Barro G., García-Dabó C. E., Gallego J., Zamorano J., 2010, ApJ, 710, 1170

López-Sanjuan C. et al., 2011, A\&A, 530, A20
Lotz J. M., Jonsson P., Cox T. J., Primack J. R., 2008, MNRAS, 391, 1137

Marconi A., Hunt L. K., 2003, ApJ, 589, L21

Martin D. C. et al., 2005, ApJ, 619, L1

Mateos S. et al., 2012, MNRAS, 426, 3271

Mazzarella J. M., Boroson T. A., 1993, ApJS, 85, 27

Mazzarella J. M., Bothun G. D., Boroson T. A., 1991, AJ, 101, 2034

McConnell N. J., Ma C.-P., 2013, ApJ, 764, 184

McGurk R. C., Max C. E., Rosario D. J., Shields G. A., Smith K. L., Wright S. A., 2011, ApJ, 738, L2

McLeod B. A., Bernstein G. M., Rieke M. J., Weedman D. W., 1998, AJ, 115, 1377

Mezcua M., Chavushyan V. H., Lobanov A. P., León-Tavares J., 2012, A\&A, 544, A36

Mezcua M., Farrell S. A., Gladstone J. C., Lobanov A. P., 2013a, MNRAS, 436, 1546

Mezcua M., Lobanov A. P., 2011, Astron. Nachr., 332,379

Mezcua M., Lobanov A. P., Chavushyan V. H., León-Tavares J., 2011, A\&A, 527, A38

Mezcua M., Roberts T. P., Sutton A. D., Lobanov A. P., 2013b, MNRAS, 436, 3128

Naab T., Johansson P. H., Ostriker J. P., 2009, ApJ, 699, L178

Oke J. B., Gunn J. E., 1983, ApJ, 266, 713

Oser L., Naab T., Ostriker J. P., Johansson P. H., 2012, ApJ, 744, 63

Phillips K. J. H., 1995, Science, 268, 1640

Ramos Almeida C., Pérez García A. M., AcostaPulido J. A., Rodríguez Espinosa J. M., 2007, AJ, 134, 2006

Roland J., Britzen S., Kudryavtseva N. A., Witzel A., Karouzos M., 2008, A\&A, 483, 125

Sanders D. B., Soifer B. T., Elias J. H., Neugebauer G., Matthews K., 1988, ApJ, 328, L35 
Schawinski K., Dowlin N., Thomas D., Urry C. M., Edmondson E., 2010, ApJ, 714, L108

Schlegel D. J., Finkbeiner D. P., Davis M., 1998, ApJ, 500, 525

Sheen Y.-K., Yi S. K., Ree C. H., Lee J., 2012, ApJS, 202, 8

Shlosman I., 2012, Proceedings of the XXIII Canary Islands Winter School of Astrophysics on 'Secular Evolution of Galaxies', Cambridge University Press (arxiv:1212.1463)

Springel V., Di Matteo T., Hernquist L., 2005, MNRAS, 361, 776

Stern D. et al., 2012, ApJ, 753, 30

Toomre A., Toomre J., 1972, ApJ, 178, 623

Trujillo I. et al., 2006, ApJ, 650, 18

van Dokkum P. G., 2005, AJ, 130, 2647

Volonteri M., Haardt F., Madau P., 2003, ApJ, 582,559

Volonteri M., Madau P., 2008, ApJ, 687, L57

Wandel A., 2002, ApJ, 565, 762

Webb N. et al., 2012, Science, 337, 554

Woo J.-H., Cho H., Husemann B., Komossa S., Park D., Bennert V. N., 2014, MNRAS, 437, 32

Wright E. L. et al., 2010, AJ, 140, 1868

This 2-column preprint was prepared with the AAS LATEX macros v5.2. 
TABle 1

Double nuCleus galaXies

\begin{tabular}{|c|c|c|c|c|c|c|c|c|c|c|}
\hline Name & $\begin{array}{c}D_{\mathrm{L}} \\
(\mathrm{Mpc}) \\
(2)\end{array}$ & $\begin{array}{c}m_{\mathrm{r}, \text { host }} \\
(3)\end{array}$ & $\begin{array}{c}\log L_{\mathrm{host}} \\
\left(\operatorname{erg~s}^{-1}\right) \\
(4)\end{array}$ & $\begin{array}{c}m_{\mathrm{r}, 1} \\
(5)\end{array}$ & $\begin{array}{r}m_{\mathrm{r}, 2} \\
(6)\end{array}$ & $\begin{array}{c}\log L_{1} \\
\left(\operatorname{erg~s}^{-1}\right) \\
(7)\end{array}$ & $\begin{array}{c}\log L_{2} \\
\left(\underset{\left.\operatorname{erg~s} \mathrm{s}^{-1}\right)}{(8)}\right.\end{array}$ & $\begin{array}{c}r \\
\left({ }^{\prime \prime}\right) \\
(9)\end{array}$ & $\begin{array}{c}r \\
(\mathrm{kpc}) \\
(10)\end{array}$ & $\begin{array}{r}\text { Hosts } \\
(11)\end{array}$ \\
\hline MCG +00-04-098 & 228 & 22.98 & 40.88 & 22.98 & 24.08 & 40.88 & 40.44 & 2.1 & 2.1 & 1 \\
\hline MCG +00-12-073 & 68 & 21.14 & 40.57 & 23.28 & 23.19 & 39.71 & 39.75 & 17.1 & 5.5 & 2 \\
\hline MCG +00-27-041 & 83 & 25.19 & 39.12 & 25.84 & 27.26 & 38.86 & 38.29 & 7.1 & 2.7 & 2 \\
\hline MCG +01-02-017 & 173 & 22.92 & 40.66 & 24.19 & 25.12 & 40.15 & 39.78 & 8.2 & 6.4 & 1 \\
\hline MCG +01-02-045 & 67 & 24.09 & 39.37 & 24.78 & 26.24 & 39.09 & 38.51 & 4.9 & 1.5 & 1 \\
\hline MCG +01-32-049 & 18 & 21.34 & 39.30 & 26.52 & 25.71 & 37.23 & 37.56 & 7.6 & 0.6 & 2 \\
\hline MCG +02-31-088 & 39 & 23.24 & 39.23 & 24.69 & 26.02 & 38.66 & 38.12 & 12.4 & 2.3 & 1 \\
\hline MCG +02-32-078 & 12 & 27.50 & 36.53 & 26.36 & 26.81 & 36.99 & 36.81 & 8.2 & 0.5 & 1 \\
\hline MCG +05-06-015 & 79 & 21.54 & 40.53 & 24.97 & 23.77 & 39.16 & 39.64 & 5.1 & 1.9 & 1 \\
\hline MCG +06-07-20 & 119 & 23.75 & 40.01 & 23.33 & 25.77 & 40.17 & 39.20 & 4.5 & 2.4 & 1 \\
\hline MCG +07-29-061 & 111 & 21.36 & 40.90 & 24.41 & 26.30 & 39.68 & 38.92 & 4.5 & 2.3 & 1 \\
\hline MCG +10-19-089 & 30 & 24.32 & 38.58 & 26.02 & 27.00 & 37.90 & 37.51 & 6.6 & 0.9 & 1 \\
\hline Mrk 19 & 59 & 21.30 & 40.38 & 23.69 & 23.86 & 39.42 & 39.35 & 4.7 & 1.3 & 1 \\
\hline Mrk 22 & 24 & 21.36 & 39.55 & 24.71 & 28.31 & 38.21 & 36.77 & 4.5 & 0.5 & $2^{\mathrm{a}}$ \\
\hline Mrk 35 & 15 & 21.34 & 39.19 & 21.56 & 22.74 & 39.10 & 38.63 & 4.0 & 0.3 & 1 \\
\hline Mrk 38 & 155 & 21.35 & 41.19 & 23.62 & 24.94 & 40.29 & 39.76 & 4.4 & 3.1 & 1 \\
\hline Mrk 66 & 89 & 22.34 & 40.32 & 24.25 & 26.06 & 39.56 & 38.83 & 5.9 & 2.4 & 1 \\
\hline Mrk 104 & 33 & 21.76 & 39.68 & 22.21 & 22.75 & 39.50 & 39.29 & 6.2 & 1.0 & 1 \\
\hline Mrk 116 & 12 & - & - & 22.45 & 23.14 & 38.56 & 38.28 & 5.4 & 0.3 & $0^{\mathrm{b}}$ \\
\hline Mrk 147 & 100 & 22.64 & 40.30 & 22.15 & 22.04 & 40.49 & 40.54 & 5.0 & 2.3 & 1 \\
\hline Mrk 153 & 36 & 21.35 & 39.92 & 22.98 & 23.84 & 39.27 & 38.92 & 4.5 & 0.8 & 2 \\
\hline Mrk 212 & 99 & 21.35 & 40.80 & 21.77 & 22.86 & 40.63 & 40.20 & 11.8 & 5.4 & 2 \\
\hline Mrk 219 & 47 & 21.08 & 40.26 & 22.85 & 23.56 & 39.55 & 39.27 & 11.4 & 2.5 & 1 \\
\hline Mrk 224 & 15 & 23.56 & 38.29 & 25.44 & 26.08 & 37.54 & 37.28 & 3.9 & 0.3 & 2 \\
\hline Mrk 306 & 73 & 21.28 & 40.57 & 23.21 & 25.00 & 39.80 & 39.08 & 4.8 & 1.6 & 1 \\
\hline Mrk 365 & 68 & 19.76 & 41.11 & 21.32 & 24.13 & 40.49 & 39.36 & 7.2 & 2.3 & 1 \\
\hline Mrk 423 & 140 & 21.34 & 41.11 & 22.09 & 24.86 & 40.81 & 39.70 & 9.2 & 5.9 & 2 \\
\hline Mrk 544 & 94 & 22.47 & 40.31 & 23.15 & 23.51 & 40.04 & 39.90 & 5.5 & 2.4 & 1 \\
\hline Mrk 553 & 58 & 21.34 & 40.34 & 26.06 & 25.65 & 38.45 & 38.62 & 7.3 & 2.0 & 2 \\
\hline Mrk 712 & 68 & 21.30 & 40.50 & 22.89 & 24.29 & 39.87 & 39.31 & 4.9 & 1.6 & 1 \\
\hline Mrk 721 & 140 & 22.24 & 40.75 & 23.83 & 23.74 & 40.11 & 40.15 & 5.4 & 3.4 & 1 \\
\hline Mrk 729 & 186 & 21.35 & 41.35 & 22.46 & 23.00 & 40.91 & 40.69 & 6.2 & 5.1 & 1 \\
\hline Mrk 731 & 25 & 21.32 & 39.62 & 22.84 & 22.51 & 39.01 & 39.14 & 4.0 & 0.5 & 2 \\
\hline Mrk 777 & 134 & 22.74 & 40.51 & 24.76 & 26.70 & 39.70 & 38.93 & 4.6 & 2.8 & 1 \\
\hline Mrk 789 & 137 & 21.30 & 41.11 & 22.21 & 23.35 & 40.74 & 40.29 & 4.7 & 2.9 & 1 \\
\hline Mrk 799 & 42 & 21.39 & 40.05 & 22.42 & 23.26 & 39.64 & 39.30 & 20.8 & 4.2 & 1 \\
\hline Mrk 930 & 71 & 22.21 & 40.18 & 22.06 & 22.73 & 40.24 & 39.97 & 4.8 & 1.6 & 1 \\
\hline Mrk 1114 & 137 & 21.32 & 41.10 & 23.43 & 24.70 & 40.26 & 39.75 & 4.2 & 2.6 & 1 \\
\hline
\end{tabular}


TABLE $1-$ Continued

\begin{tabular}{lcccccccccc}
\hline \hline Name & $\begin{array}{c}D_{\mathrm{L}} \\
(\mathrm{Mpc}) \\
(2)\end{array}$ & $\begin{array}{l}m_{\mathrm{r}, \mathrm{host}} \\
(3)\end{array}$ & $\begin{array}{c}\log L_{\mathrm{host}} \\
\left(\mathrm{erg} \mathrm{s}^{-1}\right) \\
(4)\end{array}$ & $\begin{array}{l}m_{\mathrm{r}, 1} \\
(5)\end{array}$ & $\begin{array}{c}m_{\mathrm{r}, 2} \\
(6)\end{array}$ & $\begin{array}{c}\log L_{1} \\
\left(\mathrm{erg} \mathrm{s}^{-1}\right) \\
(7)\end{array}$ & $\begin{array}{c}\log L_{2} \\
\left(\mathrm{erg} \mathrm{s}^{-1}\right) \\
(8)\end{array}$ & $\begin{array}{c}r \\
\left({ }^{\prime \prime}\right) \\
(9)\end{array}$ & $\begin{array}{c}r \\
(\mathrm{kpc}) \\
(10)\end{array}$ & $\begin{array}{c}\text { Hosts } \\
(11)\end{array}$ \\
\hline Mrk 1134 & 66 & 21.11 & 40.54 & 24.09 & 26.40 & 39.35 & 38.43 & 2.7 & 0.8 & 2 \\
Mrk 1230 & 26 & 23.60 & 38.76 & 23.59 & 24.42 & 38.76 & 38.43 & 2.2 & 0.3 & 1 \\
Mrk 1263 & 25 & 21.31 & 39.61 & 25.57 & 25.04 & 37.90 & 38.12 & 7.2 & 0.8 & 2 \\
Mrk 1307 & 20 & 21.34 & 39.40 & 23.85 & 24.69 & 38.40 & 38.06 & 8.3 & 0.8 & 2 \\
Mrk 1341 & 21 & 21.25 & 39.49 & 22.58 & 23.80 & 38.96 & 38.47 & 14.4 & 1.4 & 1 \\
Mrk 1431 & 93 & 22.12 & 40.45 & 24.25 & 25.72 & 39.59 & 39.01 & 4.8 & 2.1 & 1 \\
NGC 3049 & 25 & 21.28 & 39.63 & 21.84 & 24.14 & 39.40 & 38.48 & 2.8 & 0.3 & 1 \\
NGC 3758 & 130 & 21.32 & 41.05 & 21.51 & 23.09 & 40.98 & 40.35 & 6.2 & 3.7 & 2 \\
NGC 3773 & 18 & 21.08 & 39.45 & 22.18 & 22.32 & 39.01 & 38.95 & 3.2 & 0.3 & 1 \\
NGC 4509 & 17 & 21.36 & 39.25 & 23.92 & 26.27 & 38.23 & 37.29 & 8.3 & 0.7 & 2 \\
NGC 5058 & 17 & 21.30 & 39.31 & 24.80 & 25.37 & 37.91 & 37.68 & 2.5 & 0.2 & 2 \\
NGC 5256 & 119 & 21.35 & 40.96 & 22.44 & 23.57 & 40.53 & 40.08 & 10.2 & 5.6 & 2 \\
NGC 5860 & 77 & 21.34 & 40.59 & 27.50 & 24.02 & 38.12 & 39.51 & 9.1 & 3.3 & 2 \\
NGC 7468 & 24 & 21.39 & 39.54 & 23.35 & 24.45 & 38.76 & 38.32 & 7.7 & 0.9 & 1 \\
\hline
\end{tabular}

Note.-(1) Object name; (2) luminosity distance (from NED); (3 and 4) $r$-band apparent magnitude and luminosity, respectively, of the primary host galaxy from the photometric fit; (5 and 6 ) $r$-band apparent magnitude of primary and secondary nucleus, respectively, from the photometric fit; $(7$ and 8$) r$-band primary and secondary nuclear luminosity, respectively; (9 and 10) projected separation in arcsec and kpc, respectively; (11) number of disk host galaxies fitted. ${ }^{a}$ A de Vaucouleurs profile is needed to fit the host. ${ }^{b}$ No galaxy disk is fitted, which can be explained by the morphology of the host galaxy as a blue compact irregular (I Zw 18). 